\title{
Process control and optimization of wastewater treatment plants using simulation softwares: a review
}

\author{
Renu*, Madhu Agarwal, Kailash Singh, R.K. Dohare and S. Upadhyaya \\ Department of Chemical Engineering, Malaviya National Institute of Technology, Jaipur, India
}

\section{(C)2016 ACCENTS}

\begin{abstract}
Process control is an important part of wastewater treatment plants which is important for a plant to work at optimum conditions because it enhances lifetime of a plant and also reduce unit product cost. Process control may be as simple as on/off valve that is triggered upon offset of the system measured parameters from the desired set point. Different controllers are used in wastewater treatment plants such as combination of proportional, integral and derivative systems and also proportional/integral controls. Selection of controller is on the basis of high stability, fast response and also minimum disturbance to the system. This review is presented for commonly used and newly developed control and instrumentation in reverse osmosis and multistage flash distillation. This review paper introduces the control systems and integrated process simulation environment software used for optimization of wastewater plants.
\end{abstract}

\section{Keywords}

Wastewater, Process control, Simulation, Water.

\section{Introduction}

Among various technologies available for potable water production, the treatment of wastewater is an established technology in several countries such as the USA, Persian Gulf and European countries [1]. On the basis of working energy principle, desalination processes are classified into two classes namely thermal processes which involves phase change due to addition of heat and also membrane processes that involve pressure energy. Further thermal processes can be classified into multi-effect evaporation (MEE), multi stage flash (MSF) and vapor-compression (VC) processes. Membrane processes can be classified into $\mathrm{RO}$ and electrodialysis (ED) processes. Among these different technologies for wastewater treatment, MSF processes has many features such as it is large scale operation and has ability to deliver good quality potable water [2]. Water treatment operation was started during early part of 20th century. Water treatment is a complex process which requires efficient and accurate control system in plant to maintain operation at optimum condition so that it will cause minimum product cost and prevention of scale formation. Control systems are of two types one is conventional strategies and other one is advanced control.

*Author for correspondence

145
In conventional strategies there are PID, ratio cascade and feed forward while in advanced control it includes time delay compensation, override controller, dead time compensation etc. There are some external disturbances in a plant, these disturbances are measured by sensors and they are temperature, pressure, flow rate, $\mathrm{pH}$ and concentration. Then a signal is transferred to a controller and then it takes correct action of open or close of a valve. Then the corrective action should bring the system back to the set point.

\section{Processes used in wastewater industry}

2.1 Reverse osmosis process

Reverse Osmosis (RO) is a process which is used for production fresh water. Reverse osmosis process is capable of producing fresh water by applying high pressure into feed side surrounded by a semipermeable membrane. The applied pressure has to be larger than the osmotic pressure. The fresh water production rate is dependent on the concentration salt present in in the feed water and on membrane properties example selectivity and permeability [3]. Figure 1 shows reverse osmosis plant setup for wastewater treatment. The reverse osmosis process includes feed and product treatment system, membrane module, feed pumps and energy recovery turbines. RO process can be described as following. First of all, feed water is treated chemically and 
mechanically to reduce deposition of dust, mud and compounds such as magnesium and calcium and fouling material, non-condensable gases comes out and they require desecration. Water pressure is maintained using feed pump and then this high pressure feed stream enters in the membrane, fresh water passes through the membrane while dissolved salts are rejected by the membrane. High pressure energy of brine is recovered by turbines and product water is then treated to maintain its $\mathrm{pH}$ to neutrality [4]. The energy demand for wastewater treatment was found to be higher by $41 \%$ for multistage and
$13.3 \%$ for single stage treatment. This was achieved by continuous research and development of fouling resistant membrane and energy efficient electrical and flow equipment. Concentration polarisation and membrane fouling, both problems encountered in reverse osmosis process. These factors reduce permeate flux and productivity loss. There is requirement of suitable control system so that it is operated in cyclic manner and performance is maximized [5].

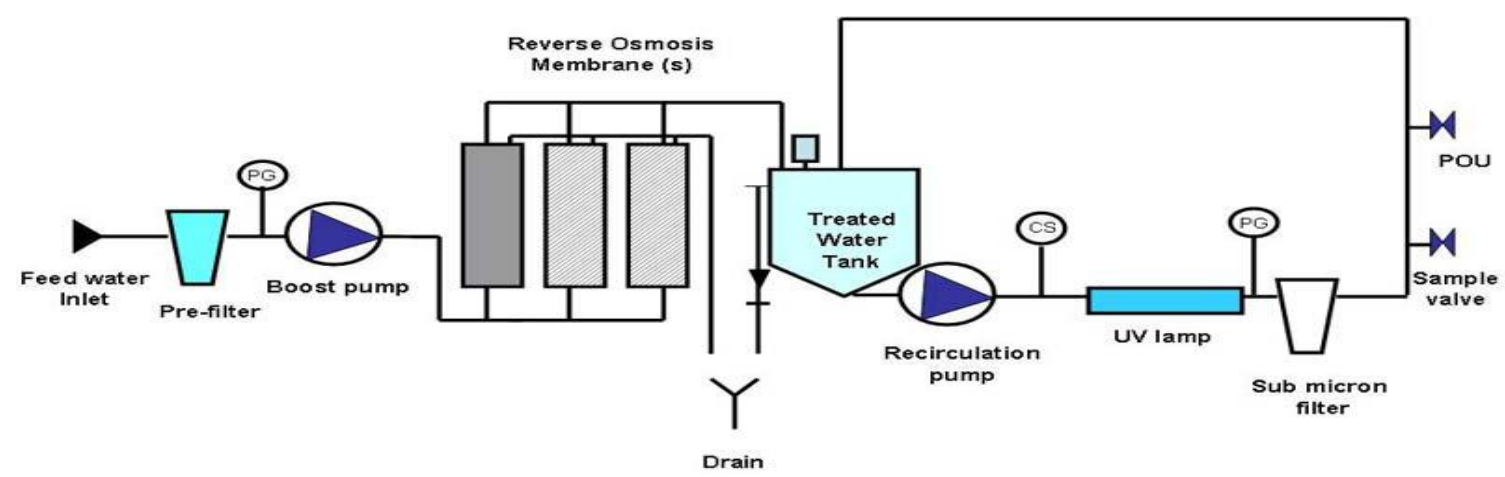

Figure 1 Reverse osmosis plant setup for wastewater treatment

\subsection{Control loops in $\mathrm{RO}$ process}

Figure 2 shows elements of a control system. Following control loops has been designed by different researchers for Reverse Osmosis process. In control loop of reverse osmosis process the main objective is to maintain a constant product rate with acceptable purity. Authors has used on/off system in reverse osmosis process and Alatiqi et al., 1999 has used first multi loop control loop which has one pressure controller and two $\mathrm{pH}$ controller. The first control loop is for feed $\mathrm{pH}$ and controlled by measuring $\mathrm{pH}$ of the feed while the second loop is for feed pressure where the measured pressure of the feed stream and permeate flux both adjust the valve. In case if there is decrement in permeate flux below the desired set point then a signal is send to the valve which increases feed pressure and it causes increases in driving force for permeation. Ali et al., 2010 has used model predictive control algorithm for reverse osmosis and they have considered two output variables such as permeate flow rate and concentration and manipulated variables includes feed pressure and brine flow rate. This control strategy is more realistic and also closer to the industrial practice. This model predictive control technique is better as compared to the PI controller because of several advantages such as good robustness characteristics and the change is adequate even if there are considerable changes in parameters of system. Dynamic Matrix Control is a predictive control technique. It is first developed at the Shell oil company [6].The DMC approach has the ability to allow plant with operation with various permeate flux, without affecting overall operation and also product quality. The model controls five system parameters such as $\mathrm{pH}$, feed temperature, flux, permeate pressure and permeate conductivity [4]. However, Abbas, 2006 has used dynamic matrix control algorithm for controlling reverse osmosis unit on a hollow fiber membrane. Author has considered two cases where in first case the permeate flux was controlled by manipulation of feed pressure and in second case both the permeate flow rate and conductivity were controlled by adjusting the feed pressure and pH. Similarly Robertson et al., 1996[7] has also used the Dynamic Matrix Control algorithm for the control of a reverse osmosis (RO) wastewater treatment pilot plant and two output variables were selected such as production flow rate and product water electrical conductivity. However Bartman et al. 2009[8] has used model-predictive control algorithm to a high capacity reverse-osmosis (RO) process with the utilisation feed flow reversal. The authors developed a dynamic nonlinear model that was used 
for simulation and Closed-loop simulations showed the ability of nonlinear model-predictive control to induce transition from high to low flow steady states in an optimal way while subjected to plant-model mismatch.

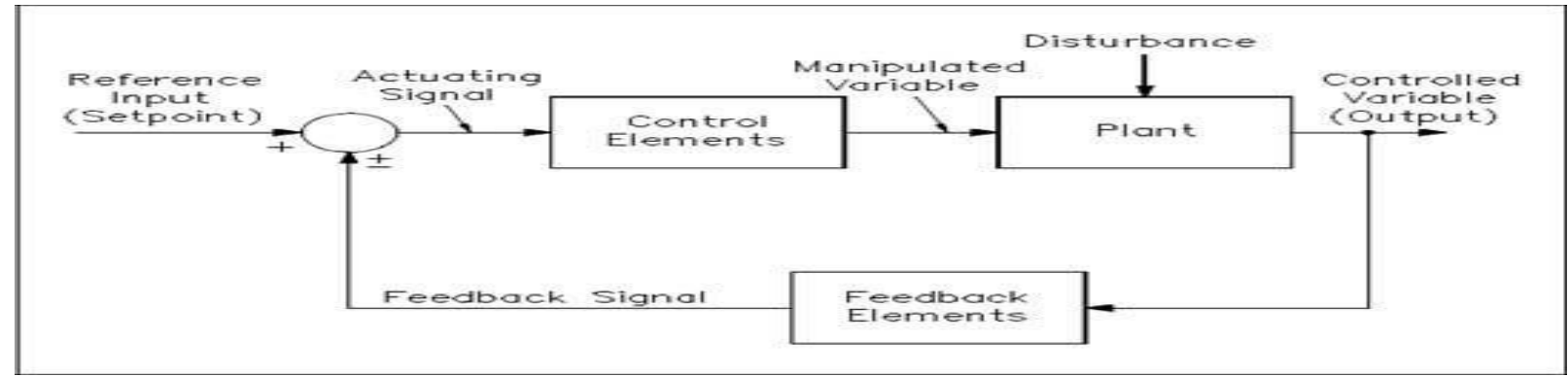

Figure 2 Elements of a control system

\subsection{Simulation techniques used for reverse osmosis process}

In Reverse osmosis process, IPSE (integrated process simulation environment) software suite is used to define simulation equations for different module, and further to connect those modules using a graphical interface. This software gives simple and rapid simulation. This software is open ended that means any equation can be defined by developer. ILF has been developed modules appropriate to the wastewater treatment processes, including reverse osmosis. The purpose behind development of wastewater treatment modules in the IPSE software is to allow simulation of different components in one simulation environment and not having to transfer data from one simulation tool to another[9] [10].

\subsection{Multi-stage flash distillation (MSF) process}

Wastewater treatment plays an important role in fresh water supply all over the world because of scarcity of water. More than $56 \%$ of the total fresh water is produced by wastewater treatment technologies under MSF process. Large scale plants adopt this process. Thus, the improvement in their reliability and efficiency avoiding possible material degradation and damages are a very important issue to maintain the water costs at an acceptable. Nowadays thermal distillation process is very popular for production of drinking process, this process converts brackish water or wastewater into potable water and the steam is provided from the turbine and generators. Vapor compression, multi-effect distillation and multistage flash distillation are some conventional thermal treatment methods in which the MSF system is more common than the other systems. $65 \%$ wastewater treatment plant in the world uses MSF technique [11]. Figure 3 shows Multi-stage flash distillation (msf) plant setup for wastewater treatment. The MSF process consists of flashing stage, vaccum system, brine heater, control loops and pumping units.

\subsection{Control loops in multi- stage flash distillation} (MSF) process

Following control loops has been designed by different researchers forMulti- stage flash distillation $(M S F)$ process: For development of a successful control system there is requirement of a proper definition of control system and a dynamic model on which we can perform designing, analysis and evaluation. For MSF several dynamics model were found in the literature. In MSF the preheated brine which leaves the last evaporator is heated in the brine heater until the saturation temperature is reached but also avoids the formation of scale in the heater. The control loop of MSF process has single input and single output system. Tayyebi et al., 2014[11] has proposed a neural network inverse model based control strategy for the MSF plant and then performance of MSF plant has been demonstrated by applying it for multistep set point tracking when the disturbance is present and then he has validated the model. Kotb et al., 2014 has used MSF model was used to design a MSF unit and feasibility of using MSF plant was found in proximity of a $650 \mathrm{MW}$ power plant. El saie and hafez., 1994 has used conventional PID controllers which were tuned to their minimum integral absolute error for disturbances regulation in the plant. Performance of plant was measured by introducing a step change inthe set point of controller during load changes. Woldai et al., 1996[12] has simulated a plant with 18 stages. The model used a speed up flow sheet and containing 153 variables, six inputs and six outputs and it was found that fixed controllers are not suitable for this MSF plant thus author has proposed to use conventional PID controllers. 


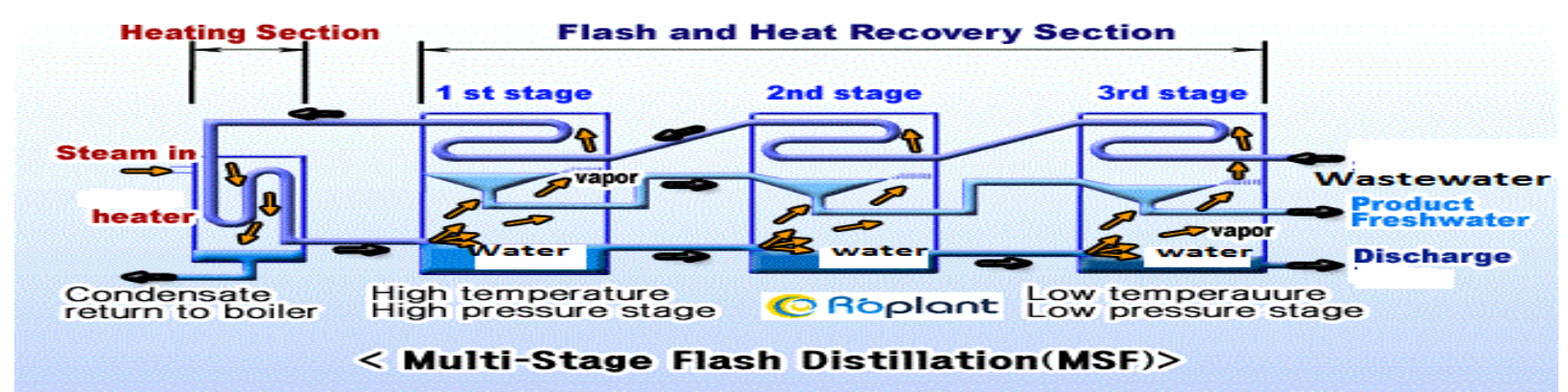

Figure 3 Multi-stage flash distillation (msf) plant setup for wastewater treatment

2.6Simulation techniques used for multi-stage flash distillation (MSF) plant

Gambier et al., 2004[13] has used SPEEDUP for simulation of MSF plant. This is a domain specific software package for chemical processing engineering. That why this is not available for control engineers. Specific software has been developed for simulation of MSF plant using programme language. Commercial software are used such as MATLAB, SIMULINK and MODELICA/DYMOLA. Using these software's models for different plants can be constructed by adding, removing, customizing pre constructed module and enabling reuse of modelling knowledge. To simulate the demisters EulerianLagrangian model was developed. This model was used for flow of water vapour simulation and brine droplets in demister.

\section{Conclusion}

Following conclusion were carried out from this review paper.

- Current practice adopts use of PID and PI controllers in wastewater treatment plants

- Dynamic matrix control provides better control than PI control thus they are suitable to use in wastewater treatment plants

- Reverse osmosis process and multistage flash distillation process both are efficient for large scale wastewater treatment with high flux and rejection over long operational periods.

- Reverse osmosis process and multistage flash distillation processes offers the low water cost and they are the best selection for wastewater treatment if fuel price are increased.

\section{Acknowledgment}

The authors wish to thanks, department of chemical engineering, MNIT Jaipur for the financial support to carry out my research work.

\section{Conflicts of interest}

The authors have no conflicts of interest to declare.

\section{References}

[1] Hinkebein TE, Price MK. Progress with the desalination and water purification technologies US roadmap. Desalination. 2005; 182(1):19-28.

[2] Bandi CS, Uppaluri R, Kumar A. Global optimization of MSF seawater desalination processes. Desalination. 2016; 394:30-43.

[3] Anqi AE, Alkhamis N, Oztekin A. Computational study of desalination by reverse osmosis-threedimensional analyses. Desalination. 2016; 388:38-49.

[4] Alatiqi I, Ettouney H, El-Dessouky H. Process control in water desalination industry: an overview. Desalination. 1999; 126(1):15-32.

[5] Ali MA, Ajbar A, Ali E, Alhumaizi K. Robust modelbased control of a tubular reverse-osmosis desalination unit. Desalination. 2010; 255(1):129-36.

[6] Cutler CR, Ramaker BL. Dynamic matrix control?? A computer control algorithm. In joint automatic control conference 1980 (p. 72).

[7] Robertson MW, Watters JC, Desphande PB, Assef JZ, Alatiqi IM. Model based control for reverse osmosis desalination processes. Desalination. 1996; 104(1):5968.

[8] Bartman AR, McFall CW, Christofides PD, Cohen Y. Model-predictive control of feed flow reversal in a reverse osmosis desalination process. Journal of Process Control. 2009; 19(3):433-42.

[9] Verhuelsdonk M, Attenborough T, Lex O, Altmann T. Design and optimization of seawater reverse osmosis desalination plants using special simulation software. Desalination. 2010; 250(2):729-33.

[10] Kotb OA. Optimum numerical approach of a MSF desalination plant to be supplied by a new specific 650MW power plant located on the Red Sea in Egypt. Ain Shams Engineering Journal. 2015; 6(1):257-65.

[11] Tayyebi S, Alishiri M. The control of MSF desalination plants based on inverse model control by neural network. Desalination. 2014; 333(1):92-100.

[12] Woldai A, Al-Gobaisi DM, Dunn RW, Kurdali A, Rao GP. An adaptive scheme with an optimally tuned PID controller for a large MSF desalination plant. Control Engineering Practice. 1996; 4(5):721-34. 
[13] Gambler A, Badreddin E. Dynamic modelling of MSF plants for automatic control and simulation purposes: a survey. Desalination. 2004; 166:191-204.

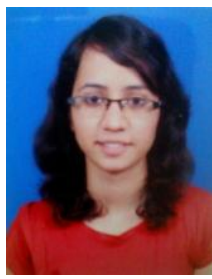

Renu is a research scholar at Malviya national institute of technology, Jaipur (302017), Rajasthan. Her date of birth is $31 / 01 / 1992$. Currently she is working on "Modelling and Experimental study of heavy metal removal from wastewater". In 2014 she has obtained M.Tech degree in chemical engineering (Process modelling and simulation) from Aligarh muslim university, Aligarh. In 2012 she has obtained B.Tech degree in chemical engineering from MJP Rohilkhand university, Bareilly. She is also a member of IIChE (Indian Institite Of Chemical Engineers), India and Asian PGPR Society of sustainable agriculture, USA.

Email:renubisht123@gmail.com

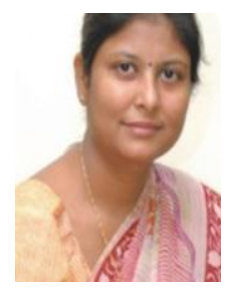

Dr. Madhu Agarwal pursued her B.Tech and M.Tech in Chemical Engineering from Calcutta University, Kolkata and Ph.D. from Malviya national institute of technology, Jaipur (302017). Currently, she is working as Assistant Professor in Department of Chemical Engineering of Malviya national institute of technology, Jaipur(302017), Rajasthan.

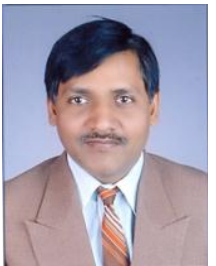

Dr. Kailash Singh pursued his B.E. from University of Roorkee, M. Tech. from IIT Kanpur and Ph.D. from Curtin University of Technology, Australia. Currently, he is working as Associate Professor in Department of Chemical Engineering of Malviya national institute of technology, Jaipur (302017), Rajasthan.

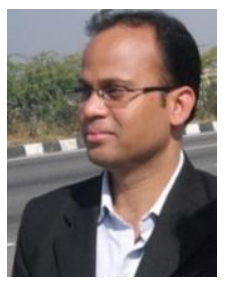

Dr. Rajeev Kumar Dohare pursued his B. Tech. in Chemical Engineering from REC Surat, M.Tech. in Chemical Engineering from AMU Aligarh and Ph.D. in Chemical Engineering from MNIT Jaipur. Currently, he is working as Assistant Professor in Department of Chemical Engineering of Malviya national institute of technology, Jaipur (302017), Rajasthan.

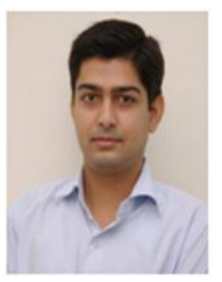

Dr. Sushant Upadhyaya pursued his B.Tech in Chemical Engineering from UPTU, Lucknow, M.Tech. in Chemical Engineering from UPTU, Lucknow and $\mathrm{Ph} . \mathrm{D}$. in Chemical Engineering from MNIT, Jaipur. Currently, he is working as Assistant Professor in Department of Chemical Engineering of Malviya national institute of technology, Jaipur(302017), Rajasthan. 